\title{
A general method for calculating the uncertainty of virtual sensors for packaged air conditioners
}

\begin{abstract}
Virtual sensors use data from low-cost measurements and calibrated models to provide outputs that would either be too expensive or impossible to measure directly. Virtual sensor technology has the potential to enable cost-effective implementation of advanced monitoring, diagnostic, and/or control features for buildings. While it is commonly known that the reliability of virtual sensors depends on the amount and conditions of calibration data, no methods have been presented that quantify the effect of the conditions of calibration data on virtual sensor output uncertainty. In this paper, a general method is presented for estimating the virtual sensor output uncertainty in terms of the uncertainty, conditions and amount of calibration data. The method is demonstrated with a power consumption virtual sensor for packaged air conditioning systems.

\section{Highlights}

- Virtual sensor uncertainty from model inputs, outputs, calibration and regression

- Virtual sensors for power consumption of packaged air conditioning units

- Effects of calibration data on virtual sensor uncertainty
\end{abstract}

\section{Keywords}

Virtual sensor; Uncertainty; Leverage; Regression diagnostics; Packaged air conditioners

\section{Nomenclature}

$\begin{array}{ll}c_{p} & \text { Specific heat at constant pressure [kJ kg-1 K-1] } \\ c_{v} & \text { Specific heat at constant volume [kJ kg-1 K-1] } \\ E & \text { Energy consumption [kWh] } \\ \boldsymbol{H} & \text { Hessian matrix [varies] } \\ \boldsymbol{J} & \text { Jacobian matrix [varies] } \\ k & \text { Specific heat ratio } \\ m & \text { Number of coefficients } \\ n & \text { Number of data points } \\ P & \text { Pressure [kPa] } \\ T & \text { Temperature [K] } \\ t_{D O F, 1-\alpha} & \text { t statistics under a confidence interval } 100(1-\alpha) \% \text { and a degree of } \\ \dot{W} & \text { freedom DOF }\end{array}$


$\vec{x}$

$\bar{x}$

$x_{i j}$

$\vec{y}$

$\hat{y}$

Greek

$\beta$

$\gamma$

$\Delta x$

$\varepsilon$

$\theta$

$\mu$

$\sigma_{x}$

$\sigma_{y x}$

$\tau$

Subscript

comp

comp+cond

cond

cov

dev

evap

ideal input

in

input

out

output

overall

train

v
Input vector [varies]

Sample mean [varies]

The entry in the $\mathrm{i}^{\text {th }}$ row and $\mathrm{j}^{\text {th }}$ column of matrix $\mathbf{X}$

Output vector [varies]

Predicted value of variable $y$ at a single data point [varies]

Coefficients [varies]

Jacobian leverage [varies]

Uncertainty of variable $x$ [varies]

Random error [varies]

Objective function [kW^2]

Population mean [varies]

Sample standard deviation of variable $\mathrm{x}$ [varies]

Mean square error of predicted variable of $y$ at input $x$ [varies]

time $[\mathrm{h}]$

\section{Introduction}

Packaged air conditioning units, such as rooftop units (RTUs), provide cooling for over $40 \%$ of the air-conditioned floor space in commercial buildings in the United States (EIA 2003).

Furthermore, space cooling is responsible for over $14 \%$ of the total primary energy use in commercial buildings in (DOE 2007). As a result, new technologies that can improve the overall operational efficiency of RTUs, such as advanced diagnostics or optimal control, have received increasing attention. However, some of the techniques require expensive measurements such 
as power and capacity, which has hindered their widespread adoption. To reduce the cost of the measurement, virtual sensors for RTUs have been proposed and evaluated.

Virtual sensing technology estimates some system quantity of interest using a regression model and low-cost measurements. A variety of virtual sensors have been developed in recent years for different HVAC equipment to replace measurements that are either expensive or impossible to measure directly. For instance, airflow rate can be measured directly with hot wire anemometry but it is too expensive for widespread application. On the other hand, a virtual air flow sensor could employ a fan-motor characteristic along with a differential pressure measurement as an input. In a general sense, a virtual sensor uses a regression model to predict the required output quantity using inexpensive sensors to provide the necessary inputs.

Li et al. (2011) gave an extensive review of different types of virtual sensors that included virtual refrigerant charge, refrigerant pressure, virtual refrigerant flow rate, and virtual compressor power consumption sensors for vapor compression equipment. Recent developments can also be found in Yu et al. (2011a, b) for virtual supply air temperature and airflow sensors for RTUs, in Song et al. (2012) for virtual water flow rate measurement, in Zhao et al. (2012) for a virtual condenser fouling sensor and in Kim and Braun (2012) for improved virtual refrigerant charge sensors.

All physical measurements require specification of accuracy and uncertainty, and virtual sensors should also meet this requirement. Song et al. (2012) and Yu et al. (2011b) discussed how measurement uncertainties in inputs to virtual sensors propagate to their outputs. Song et al. (2011) considered how the uncertainty due to the mean squared error of the regression model and the uncertainty from calibration data in the regression process affect the uncertainty of the virtual sensor output. However, the effects of the uncertainty of the regression model on the virtual sensor output were not considered. In addition, existing uncertainty calculation methods only estimate uncertainty between virtual sensor predictions and values obtained by direct measurement of the predicted variable. These uncertainties are not directly comparable with those of the direct measurements because they are not referenced to the true values of the quantities.

In this paper, an uncertainty calculation approach is developed and demonstrated that can estimate virtual sensor uncertainties relative to true values so that overall virtual sensors can be evaluated in terms of accuracy and cost relative to direct measurement approaches. The method considers the uncertainty due to time fluctuation of sensor readings, the uncertainty of the physical sensors, and the uncertainty due to the regression process. Results are presented for virtual power sensors that were calibrated using field data from 3 RTUs and the dominant sources of uncertainty are identified for a variety of conditions.

This paper is divided into development and application sections. Section 2 develops a generalized virtual sensor output uncertainty approach, whereas section 3 applies the general 
approach for evaluating the uncertainty associated with virtual power sensors for RTUs. The application includes demonstration results using field data from three RTUs.

\section{Virtual Sensor Output Uncertainty Development}

An output of a virtual sensor should be an unbiased estimator of the true value of the quantity. Its uncertainty describes the probability distribution of the difference between the true value and the prediction of the quantity in a future observation. The uncertainty of a virtual sensor output is decoupled into several components related to regression model uncertainty, uncertainty in data measurements used for model calibration, uncertainty in virtual sensor input measurements and the uncertainty in the measured outputs used as the basis for evaluating the virtual sensor. This section develops uncertainty equations for each of these components but begins with a general definition and calculation approach for uncertainty.

\subsection{Uncertainty definition and calculation}

Uncertainty of a quantity describes the probability distribution of the difference between its estimated and true value. The true values of quantities are usually defined by standards. For instance, $1 \mathrm{~K}$ is defined as the fraction $1 / 273.16$ of the thermodynamic temperature of the triple point of water where $273.16 \mathrm{~K}$ is defined as the true value of the triple point of water (Taylor and Thompson 2008). In practice, measurement devices are calibrated against standards and used to estimate the true values of a quantity at any condition. To facilitate the discussion, this paper defines estimation as an action to obtain any quantities with uncertainties, measurement as an action to obtain quantities by physical measurement and prediction as an action to obtain quantities by analytical methods such as virtual sensors. In other words, both measurement and prediction are techniques to estimate true values of quantities with some uncertainties.

Measurement estimates quantities by physical sensors, and prediction estimates quantities by analytical methods.

A common source of uncertainty in estimation is the uncertainty of the measurement tools. Although it is more convenient to use the tools to obtain the quantities than to compare the quantities with the standards, they carry uncertainties and cannot estimate quantities exactly at their true values. For example, when the temperature of an object is measured, a thermocouple with a measurement uncertainty $\pm 0.5 \mathrm{~K}$ can be used, and the true value of the object lies within $\pm 0.5 \mathrm{~K}$ of the measured temperature (ASHRAE 2006).

The uncertainty also depends on the environmental noise. If an ideal tool with zero uncertainty is used to estimate a quantity subjected to environmental noise by repeated estimation over time, the time-average of the quantity will have an uncertainty due to the environmental noise. To quantify this uncertainty, a sample standard deviation shown in Eqn. (1) is calculated.

$$
\sigma_{x}=\sqrt{\frac{\sum_{i}\left(x_{i}-\bar{x}\right)^{2}}{n-1}}
$$


When the individual values in the sample carry no uncertainty, the uncertainty of the average value of the quantity can be quantified by a $100(1-\alpha) \%$ confidence interval in Eqn. (2).

$$
\Delta \bar{x}_{\text {ideal input }}=t_{n-1,1-\alpha} \sigma_{x} \sqrt{\frac{1}{n}}
$$

In some situations, it is possible to have uncertainties from different independent origins. Assuming that the distribution functions of the uncertainties are symmetrical and are independent of each other, they are summed for the overall uncertainty by the root-sumsquares method as Eqn. (3).

$$
\Delta x_{\text {overall }}=\sqrt{\sum_{i}\left(\Delta x_{i}\right)^{2}}
$$

The propagation of the uncertainty from the calibration data to the prediction output is given by Eqn. (4), which computes the uncertainty of a function $f$ propagated from its independent model inputs (Kline and McClinktock1953).

$$
\Delta f\left(x_{1}, x_{2}, \ldots, x_{i}, \ldots\right)=\sqrt{\sum_{i}\left(\frac{\partial f\left(x_{i}\right)}{\partial x_{i}} \Delta x_{i}\right)^{2}}
$$

The uncertainty of a sample mean of an estimated quantity can be derived from Eqn. (2), (3) and (4) to form Eqn. (5) (Moffat 1988).

$$
\Delta \bar{x}=\sqrt{\frac{\sum_{i}\left(\Delta x_{i}\right)^{2}}{n}+\frac{\left(t_{n-1,1-\alpha} \sigma_{x}\right)^{2}}{n}}
$$

\subsection{Regression model uncertainty}

To begin with, the uncertainty of regression models is discussed by considering a hypothetical linear regression model in Eqn. (6) with a true input vector, a true output and a vector of true coefficients (Montgomery 2005; Graybill and lyer 1994).

$$
y_{\text {true }}=\vec{x}_{\text {true }}^{T} \vec{\beta}_{\text {true }}+\varepsilon
$$

Regression is conducted using calibration data obtained from the modeled system and involves estimating a set of coefficients that minimizes the difference between the model output $y$ and the corresponding values in the calibration data. The regression model in Eqn. (6) is predicted as Eqn. (7) where a predicted model output depends on the input vector and a vector of predicted coefficients. 


$$
\hat{y}=\vec{x}^{T} \overrightarrow{\hat{\beta}}
$$

Since the model output, the model input and the model coefficients in Eqn. (6) are true for this hypothetical example, they carry zero uncertainty. However, the linear equation $\vec{x}_{\text {true }}^{T} \vec{\beta}_{\text {true }}$ may not be a perfect model, and it carries an uncertainty $\varepsilon$ to represent the difference between the model output and the true value of the quantity. To quantify the uncertainty $\varepsilon$ in Eqn. (6), the uncertainties of the estimated output and the predicted coefficients are considered. By assuming that the regression model does not have any systematic error and $\varepsilon$ represents the random error of the regression model only, the resultant expression for output uncertainty of the regression model is Eqn. (8), where the mean square error is shown in Eqn. (9) and the matrix $\mathbf{X}_{\text {train }}$ is a matrix formed by input vectors in the calibration data as shown in Eqn. (10)(Montgomery 2005).

$$
\begin{gathered}
\Delta \hat{y}=t_{n-m-1,1-\alpha} \sigma_{y x} \sqrt{1+\vec{x}^{T}\left(\mathbf{X}_{\text {train }}^{T} \mathbf{X}_{\text {train }}\right)^{-1} \vec{x}} \\
\sigma_{y x}=\sqrt{\frac{\sum_{i}\left(\hat{y}_{i}-y_{i}\right)^{2}}{n-m}} \\
\mathbf{X}_{\text {train }}=\left[\begin{array}{c}
\vec{x}_{\text {train }, 1}^{T} \\
\vdots \\
\vec{x}_{\text {train }, n}^{T}
\end{array}\right]
\end{gathered}
$$

According to Laurent and Cook (1993), $\vec{x}^{T}\left(\boldsymbol{X}_{\text {train }}^{T} \boldsymbol{X}_{\text {train }}\right)^{T} \vec{x}$ is called tangential leverage and is a measure of the influence of a calibration data point on the regression result. When $y$ is predicted using a nonlinear equation in Eqn. (11) with $\vec{x}$ and $\vec{\beta}$, then nonlinear regression is required and Eqn. (8) does not apply. In this case, a Jacobian leverage $\gamma$ and the uncertainty component are calculated by Eqns. (12), (13), (14) and (16).

$$
\begin{gathered}
\hat{y}=f(\vec{x}, \overrightarrow{\hat{\beta}}) \\
\mathbf{J}_{\text {train }}=\left[\begin{array}{c}
\left(\frac{\partial f\left(\vec{x}_{\text {train }, 1}, \overrightarrow{\hat{\beta}}\right)^{T}}{\partial \overrightarrow{\hat{\beta}}}\right)^{T} \\
\vdots \\
\left(\frac{\partial f\left(\vec{x}_{\text {train, },}, \overrightarrow{\hat{\beta}}\right)}{\partial \overrightarrow{\hat{\beta}}}\right)^{T}
\end{array}\right]
\end{gathered}
$$




$$
\begin{aligned}
\mathbf{H}_{\text {train }, i}= & {\left[\begin{array}{ccc}
\frac{\partial^{2} f\left(\vec{x}_{\text {train }, i}, \overrightarrow{\hat{\beta}}\right)}{\partial \hat{\beta}_{1}^{2}} & \ldots & \frac{\partial^{2} f\left(\vec{x}_{\text {train }, i}, \overrightarrow{\hat{\beta}}\right)}{\partial \hat{\beta}_{m} \partial \hat{\beta}_{1}} \\
\vdots & \frac{\partial^{2} f\left(\vec{x}_{\text {train }, i}, \overrightarrow{\hat{\beta}}\right)}{\partial \hat{\beta}_{j} \delta \hat{\beta}_{k}} & \vdots \\
\frac{\partial^{2} f\left(\vec{x}_{\text {train }, i}, \overrightarrow{\hat{\beta}}\right)}{\partial \hat{\beta}_{1} \partial \hat{\beta}_{m}} & \frac{\partial^{2} f\left(\vec{x}_{\text {train }, i}, \overrightarrow{\hat{\beta}}\right)}{\partial \hat{\beta}_{m}^{2}}
\end{array}\right] } \\
\gamma= & \left.\left(\frac{\partial f}{\left.\partial \overrightarrow{\hat{\beta}}_{\text {train }}-\overrightarrow{\hat{y}}_{\text {train }}\right]\left[\mathbf{H}_{\text {train }}\right]=\Sigma_{i}\left(y_{\text {train }, i}-\hat{y}_{\text {train }, i} \mathbf{J}_{\text {train }}-\left[\mathbf{H}_{\text {train }, i}\right.\right.} \mathbf{y}_{\text {train }}-\overrightarrow{\hat{\hat{y}}}_{\text {train }}\right]\left[\mathbf{H}_{\text {train }}\right]\right)^{-1}\left(\frac{\partial f}{\partial \overrightarrow{\hat{\beta}}}\right) \\
\Delta \hat{y}=t_{n-m, 1-\alpha} \sigma_{y x} \sqrt{1+\gamma} &
\end{aligned}
$$

The overall model uncertainty in Eqn. (16) is decoupled into the sum of two parts using Eqn. (3). The first part is termed the model calibration output deviation and is the uncertainty associated with model predictions at the calibration points represented by Eqn. (17).

$$
\Delta \hat{y}_{d e v}=t_{n-m, 1-\alpha} \sigma_{y x}
$$

A more accurate model results in a smaller uncertainty in the model output deviation.

The second part is termed the uncertainty from model covariance given by Eqn. (18).

$$
\Delta \hat{y}_{c o v}=t_{n-m, 1-\alpha} \sigma_{y x} \sqrt{\gamma}
$$

Since the Jacobian leverage $\gamma$ in Eqn. (18) is a measure of the confidence of the model at inputs different from the calibration data points, this uncertainty component characterizes how confidently the model describes the behavior at a variety of inputs (Allus et al. 1989). The uncertainty component grows as the confidence decreases.

\subsection{Uncertainty due to calibration data}

The regression to predict the coefficient vector $\overrightarrow{\hat{\beta}}$ in Eqn. (11) can be described as a functional relationship in terms of the calibration input and output data as shown in Eqn. (19).

$$
\overrightarrow{\hat{\beta}}=g\left(\vec{x}_{\text {train }, 1}, \vec{x}_{\text {train }, 2}, \ldots, \vec{x}_{\text {train }, n}, y_{\text {train }, 1}, y_{\text {train }, 2}, \ldots, y_{\text {train }, n}\right)
$$


The uncertainty in the calibration data propagates to the prediction output $\hat{y}$ in Eqn. (11) through the dependence of the predicted coefficient vector $\overrightarrow{\hat{\beta}}$ on the calibration data. The uncertainty propagation results in the uncertainty due to calibration data as described by Eqn. (20).

$$
\Delta \hat{y}_{\text {train }}=\sqrt{\Sigma_{l} \Sigma_{k}\left(\left(\Sigma_{p} \frac{\partial f(\vec{x}, \overrightarrow{\hat{\beta}})}{\partial \hat{\beta}_{p}} \frac{\partial \hat{\beta}_{p}}{\partial x_{l k}}\right) \Delta x_{l k, \text { train }}\right)^{2}+\Sigma_{k}\left(\left(\Sigma_{p} \frac{\partial f(\vec{x}, \overrightarrow{\hat{\beta}})}{\partial \hat{\beta}_{p}} \frac{\partial \hat{\beta}_{p}}{\partial y_{k}}\right) \Delta y_{k, \text { train }}\right)^{2}}
$$

The derivatives in Eqn. (20) that cannot be differentiated analytically are approximated by using finite differences (Nocedal and Wright 2000).

If all calibration data points carry the same uncertainty, the magnitude of Eqn. (20) decreases with increasing amount of calibration data because the dependence of the predicted regression coefficients on each calibration data point decreases with increasing number of calibration data points. Hence the uncertainty from calibration data usually decreases with an increase of the amount of calibration data.

\subsection{Uncertainty due to inputs}

As the predicted output of Eqn. (11) depends on the input vector $\vec{x}$ and each entry in the input vector carries an uncertainty, these uncertainties propagate to the predicted output. Following Eqn. (4), the uncertainty due to inputs of the prediction is described by Eqn. (21).

$$
\Delta \hat{y}_{\text {input }}=\sqrt{\sum_{i}\left(\frac{\partial f(\vec{x}, \overrightarrow{\hat{\beta}})}{\partial x_{i}} \Delta x_{i}\right)^{2}}
$$

Uncertainty due to inputs in Eqn. (21) is small when the uncertainty of the inputs is small.

\subsection{Uncertainty due to outputs}

Although the output of Eqn. (11) is arranged to predict its true value, only the calibration data are used to predict the coefficient vector $\overrightarrow{\hat{\beta}}$, and the model of Eqn. (11) is only calibrated to predict observed values of $y$ from the calibration data and not the true values of $y$. However, the output uncertainties are defined as the distribution of the differences between the virtual sensor output and its true value over all possible conditions. To include the effects of output uncertainties outside the calibration data set, the relationship between the observed value of $y$ in the calibration data and its true value is considered in Eqn. (22).

$$
y_{\text {true }}=y \pm \Delta y
$$




\subsection{Overall uncertainty}

For a virtual sensor which is mathematically described by Eqn. (11), the overall uncertainty of its output is defined as Eqn. (24) by summing all its components with Eqn. (3).

$$
\Delta \hat{y}_{\text {overall }}=\sqrt{\left(\Delta \hat{y}_{\text {cov }}\right)^{2}+\left(\Delta \hat{y}_{\text {dev }}\right)^{2}+\left(\Delta \hat{y}_{\text {train }}\right)^{2}+\left(\Delta \hat{y}_{\text {input }}\right)^{2}+\left(\Delta \hat{y}_{\text {output }}\right)^{2}}
$$

\section{Case study application to virtual RTU power outputs}

To demonstrate application of the virtual sensor output uncertainty method, power consumption virtual sensors were calibrated with data collected from 3 single-speed RTUs. Data were collected in the field at a small restaurant near Philadelphia for a few weeks from July to September in 2013. Some basic specifications of the RTUs are given in Table 1.

Table 1 Specifications of experimental setups

\begin{tabular}{|l|l|l|l|}
\hline System & RTU 2 & RTU 3 & RTU 4 \\
\hline Nominal cooling capacity $[\mathrm{kW}]$ & 14.1 & 14.1 & 17.2 \\
\hline Refrigerant & R22 & R22 & R410A \\
\hline Compressor & Reciprocating & Reciprocating & Scroll \\
\hline Condenser airflow $[\mathrm{cfm}]$ & 4000 & 4000 & 4000 \\
\hline
\end{tabular}

During cooling season the RTUs can operate in cooling mode or economizer mode. In cooling mode, the compressor, condenser fan and evaporator fan are on. In the economizer mode, only the evaporator fan operates and the compressor and condenser fan remain off. The compressor, evaporator fan, and condenser fan operate at a single speed with on/off control for the three RTUs.

\subsection{Measurement instrumentation, processing, and uncertainty}

The units were well instrumented for this study. Refrigerant temperature at compressor suction, compressor discharge, condenser return bend and evaporator inlet for each RTU were measured using T-type thermocouples with uncertainty $\pm 0.5 \mathrm{~K}$ that were surface mounted. Current switches were attached to electrical wiring of the compressor, evaporator fan and condenser fan to record their on-off statuses. Energy meters were installed to measure the total energy consumption of each RTU and yielded an electrical signal for every $0.1 \mathrm{kWh}$ of electricity 
consumed. A schematic is shown in Figure 1, where T stands for surface mounted thermocouples, $\mathrm{S}$ stands for current switches and $\mathrm{W}$ stands for an energy meter. The $\mathrm{T}$ on the condenser denotes a temperature sensor that was installed on one of the return bends along the condenser refrigerant circuit.

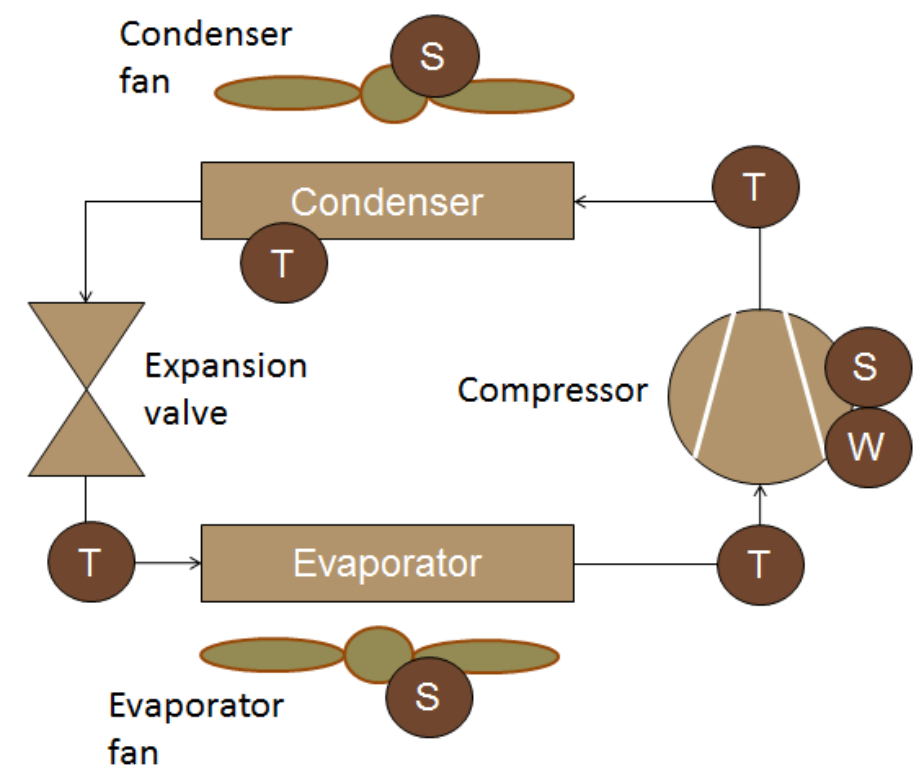

Figure 1 Schematic of sensor installation in each RTU

Data were collected every minute and data analysis involved averaging temperature readings and integrating energy meter readings over time. While each temperature reading has its own measurement uncertainty, their fluctuation about the average reading with time creates another uncertainty component. With a $95 \%$ confidence interval, the uncertainty of an average temperature over time is derived from Eqn. (5) to give Eqn. (25).

$$
\Delta \bar{T}=\sqrt{\frac{(\Delta T)^{2}}{n}+\frac{\left(t_{n-1,0.95} \sigma_{T}\right)^{2}}{n}}
$$

The average power consumption of RTUs for a certain period of time are calculated by Eqn. (26) through integration of the energy consumption.

$$
\dot{W}=\frac{\Sigma E}{\tau}
$$

The integrating power meter internally records electrical energy usage in increments of $0.1 \mathrm{kWh}$ and outputs the integrated values to the data acquisition system at the sampling interval. It is believed that the primary source of error is associated with the potential for a mismatch between the timing of the data acquisition sampling time and internal timing for incrementing 
the energy integration. With this assumption, only the uncertainty at the first and last time step of the sampling period propagates to the integrated energy and average power consumption and the uncertainty of the average power consumption can be expressed as Eqn. (27).

$$
\Delta \dot{W}=\sqrt{2}\left(\frac{\Delta E}{\tau}\right)
$$

where $\Delta \mathrm{E}$ is the uncertainty associated with the energy measurement in the first or the last step of the sampling interval. Since the energy meters update their integrals for every $0.1 \mathrm{kWh}$ of power consumption, $\Delta \mathrm{E}$ in Eqn. (27) is $0.05 \mathrm{kWh}$.

For each RTU, the evaporator fan can operate independently of the compressor to provide free cooling in economizer mode or ventilation during the occupied period in fan mode. However, the compressor and condenser fan for each unit cycle on and off together in response to calls for cooling by the thermostat. Because of this decoupled behavior, separate virtual power sensors were developed for the evaporator fan and the total power associated with the compressor and condenser fan of each RTU. The virtual power sensors described in the following subsection and appendices were calibrated with data collected during the month of August, 2013. Since only a single power measurement was installed on the unit, the evaporator fan power consumption was assumed to be the total power measurement whenever the compressor was not operating. The average fan power consumption and measurement uncertainty were calculated using Eqns. (26) and (27) with an integration period equal to the operating time of each separate economizer mode operation. For example, when the algorithm found a 10-minute economizer mode operation between two compressor operation periods, it obtained a fan power consumption value by integrating the energy consumption in the 10minute period. The data for calibration of the evaporator fan power consumption virtual sensor were filtered to only include measurements having relative measurement uncertainty lower than $5 \%$. The filtering process lowered the average relative measurement uncertainty of the fan power consumption of RTUs from $16.7 \%$ to $2.09 \%$ after removing $58.9 \%$ of the available data. The total number of evaporator fan power data points available for virtual sensor calibration applied to RTUs 2, 3 and 4 were 62, 62 and 59, respectively.

The combined compressor and condenser fan power measurements were determined as the difference between the total power measurement and the virtual evaporator fan power prediction. The steady-state detector used compressor suction temperature, compressor discharge temperature, condenser return bend temperature and evaporator inlet temperature as inputs to a method based on the approach described by Li and Braun (2007). A data point was considered to be at steady state if the following criteria were satisfied within a moving data window. 
To avoid calibrating the virtual sensors with duplicate data points, data points were grouped together by comparing average temperatures of the compressor suction, compressor discharge, condenser return bend and evaporator inlet. Data points with average temperature readings that were statistically equivalent according to the uncertainties were grouped together to form a single data point by averaging their power consumption and temperature. The uncertainty of the temperature and power consumption of the new data point was obtained using Eqn. (5). Data filtering was conducted and only data with a power consumption uncertainty value less than $5 \%$ of the power consumption and a positive compressor suction superheat were used to calibrate the sensors for RTU 2, 3 and 4. Only one data point was removed from RTU 2 data using the filtering criteria. The resultant number of data points for calibration of the virtual compressor and condenser fan power sensor for RTUs 2, 3 and 4 were 57, 80 and 72, respectively.

\subsection{Overview of virtual power sensors and uncertainty}

For each RTU, the evaporator fan can operate independently of the compressor to provide ventilation and free cooling whereas the compressor and condenser fan cycle on and off in response to calls for cooling by the thermostat. Because of this decoupled behavior, separate virtual power sensors were developed for the evaporator fan and the total power associated with the compressor and condenser fan of each RTU.

The evaporator fan power consumption virtual sensor outputs a fixed value when the fan is on that was learned using training data as described in Appendix A. Since the model is very simple and there is no uncertainty in the only input (fan on/off status), the virtual sensor output uncertainty calculation is very straightforward as presented in Appendix A. 
The virtual sensor for the compressor and condenser fan combination is much more complicated and is based on the semi-empirical model of Jähnig et al. (2000) that has inputs of suction and discharge pressure along with suction temperature. A detailed description of this model is provided in Appendix B along with application of the method of section 2 in determining the overall virtual sensor output uncertainty.

\subsection{Accuracy and reliability of the virtual power sensors}

To analyze overall accuracy and reliability of the virtual sensors over a range of conditions for the case study, coefficient of variation and average relative uncertainty were calculated. The coefficient of variation has been widely used to evaluate accuracy for a long time (Hendricks and Robey 1936; Kesteven 1946) and is defined in Eqn. (29).

$$
\text { Coefficient of variation }=\frac{\sigma_{y x}}{\bar{y}}
$$

The coefficient of variation compares the mean square error of the virtual sensor output with the mean value of the quantity from the calibration data. A small coefficient of variation implies an accurate model.

Relative uncertainty of a virtual sensor is given by Eqn. (30), and the average relative uncertainty of the virtual sensor is given by Eqn. (31).

$$
\begin{gathered}
\text { Relative uncertainty }=\frac{\Delta \hat{y}(\vec{x}, \overrightarrow{\hat{\beta}})}{\hat{y}(\vec{x}, \overrightarrow{\hat{\beta}})} \\
\text { Average relative uncertainty }=\frac{1}{n} \sum_{i} \frac{\Delta \hat{y}\left(\vec{x}_{\text {train }, i}, \overrightarrow{\hat{\beta}}\right)}{\hat{y}_{\text {train }, i}\left(\vec{x}_{\text {train }, i}, \overrightarrow{\hat{\beta}}\right)}
\end{gathered}
$$

Relative uncertainty examines if the virtual sensor is reliable given an input vector, and the average relative uncertainty examines the average reliability of the virtual sensor at its calibration data points. A small average relative uncertainty indicates that the virtual sensor outputs are not significantly affected by the random error from calibration data or regression. This means that the methodology and the sensors will not give significantly different outputs due to randomness in the inputs and calibration process, and the sensors are reliable within the conditions described by the calibration data. However, to examine the reliability of a virtual sensor with inputs that differ from calibration data, the relative uncertainty of the virtual sensor with a set of test inputs should also be examined.

To analyze the significance of any uncertainty component, uncertainties from output deviation, calibration data, covariance, inputs and outputs are normalized according to Eqn. (32). 


$$
\text { Relative uncertainty component }=\left(\frac{\text { Uncertainty component }}{\text { Overall uncertainty }}\right)^{2}
$$

The normalization equation Eqn. (32) is squared so that the uncertainty components can be summed to $100 \%$.

The virtual sensor prediction for evaporator fan power consumption is analyzed in Table 2.

Table 2 Virtual evaporator fan power sensor calibration results

\begin{tabular}{|r|r|l|l|r|r|}
\hline & & $\begin{array}{l}\text { Average } \\
\text { relative } \\
\text { uncertainty } \\
\text { of } \\
\text { measurement } \\
\text { predicted } \\
\text { power } \\
{[\mathrm{kW}]}\end{array}$ & $\begin{array}{l}\text { Average relative } \\
\text { uncertainty of } \\
\text { prediction at } \\
\text { calibration } \\
\text { scenarios }\end{array}$ & $\begin{array}{l}\text { Coefficient } \\
\text { of variation }\end{array}$ & $\begin{array}{l}\text { Number of } \\
\text { data points }\end{array}$ \\
\hline 2 & 0.78 & $2.39 \%$ & $4.41 \%$ & $1.84 \%$ & 62 \\
\hline 3 & 0.81 & $2.09 \%$ & $4.45 \%$ & $1.96 \%$ & 62 \\
\hline 4 & 0.95 & $1.77 \%$ & $3.81 \%$ & $1.68 \%$ & 59 \\
\hline
\end{tabular}

The virtual evaporator fan power sensors for RTU 2, 3 and 4 have good accuracy and reliability with a coefficient of variation smaller than $2 \%$ and a relative uncertainty of prediction smaller than $4.5 \%$ as shown in Table 2.

To examine the main source of the uncertainty, the relative uncertainties are decoupled and tabulated in Table 3.

Table 3 Relative uncertainty components of virtual evaporator fan power sensor outputs

\begin{tabular}{|r|l|l|r|r|}
\hline & $\begin{array}{l}\text { Relative } \\
\text { uncertainty from } \\
\text { model output } \\
\text { deviation }\end{array}$ & $\begin{array}{l}\text { Relative uncertainty } \\
\text { from model } \\
\text { covariance }\end{array}$ & $\begin{array}{l}\text { Relative } \\
\text { uncertainty from } \\
\text { calibration data }\end{array}$ & $\begin{array}{l}\text { Relative } \\
\text { uncertainty } \\
\text { from output }\end{array}$ \\
\hline 2 & $69.94 \%$ & $0.02 \%$ & $0.56 \%$ & $29.47 \%$ \\
\hline 3 & $77.46 \%$ & $0.02 \%$ & $0.43 \%$ & $22.09 \%$ \\
\hline 4 & $78.01 \%$ & $0.02 \%$ & $0.44 \%$ & $21.52 \%$ \\
\hline
\end{tabular}

The input uncertainty is zero for the evaporator fan power virtual sensor and therefore is not listed in Table 3. Table 3 shows that the relative uncertainty from model output deviation is the most significant uncertainty component. Since the output does not change with the environmental conditions, the uncertainty of the sensor remains constant in all scenarios after calibration.

The overall performance of the compressor and condenser fan power consumption virtual sensor and their uncertainty components are shown in Table 4 and Table 5. 
Table 4 Calibration results for virtual compressor and condenser fan power sensors

\begin{tabular}{|r|r|r|r|r|}
\hline RTU & $\begin{array}{l}\text { Average } \\
\text { estimated } \\
\text { power [kW] }\end{array}$ & $\begin{array}{l}\text { Average relative } \\
\text { uncertainty of prediction } \\
\text { at calibration scenarios }\end{array}$ & $\begin{array}{l}\text { Coefficient of } \\
\text { variation }\end{array}$ & $\begin{array}{l}\text { Number of data } \\
\text { points }\end{array}$ \\
\hline 2 & 4.07 & $4.55 \%$ & $1.51 \%$ & 57 \\
\hline 3 & 4.52 & $4.44 \%$ & $1.65 \%$ & 80 \\
\hline 4 & 3.79 & $5.27 \%$ & $1.90 \%$ & 72 \\
\hline
\end{tabular}

Table 5 Relative uncertainty components of virtual compressor and condenser fan power sensor outputs during calibration period

\begin{tabular}{|r|r|l|l|l|l|}
\hline & $\begin{array}{l}\text { Relative } \\
\text { uncertainty } \\
\text { from model } \\
\text { output } \\
\text { deviation }\end{array}$ & $\begin{array}{l}\text { Relative } \\
\text { uncertainty } \\
\text { from model } \\
\text { covariance }\end{array}$ & $\begin{array}{l}\text { Relative } \\
\text { uncertainty } \\
\text { from } \\
\text { calibration data }\end{array}$ & $\begin{array}{l}\text { Relative } \\
\text { uncertainty } \\
\text { from input }\end{array}$ & $\begin{array}{l}\text { Relative } \\
\text { uncertainty } \\
\text { from output }\end{array}$ \\
\hline 2 & $44.5 \%$ & $3.1 \%$ & $31.9 \%$ & $0.3 \%$ & $20.3 \%$ \\
\hline 3 & $55.3 \%$ & $2.6 \%$ & $24.6 \%$ & $0.3 \%$ & $17.1 \%$ \\
\hline 4 & $52.6 \%$ & $3.0 \%$ & $26.5 \%$ & $0.5 \%$ & $17.4 \%$ \\
\hline
\end{tabular}

The coefficients of variation in Table 4 are less than $2.7 \%$ and the average relative uncertainties are less than $5.3 \%$. The low coefficients of variation and uncertainties of the sensors in Table 4 imply that the sensors are accurate and reliable.

Table 5 shows that the uncertainty from model output deviation is the most significant uncertainty component. The uncertainty from calibration data and the uncertainty from output measurements are also significant in contributing to the overall uncertainty of the virtual sensor output.

\subsection{Change of uncertainty with calibration data}

To examine the effect of the number of calibration data points on virtual sensor uncertainty, the compressor and condenser fan power consumption sensors were calibrated with data collected for different time periods beginning on July 31, 2013. As the calibration period increases, the number of calibration data points increases. The virtual sensors were trained for increasing periods in increments of every two days starting from the $15^{\text {th }}$ day of data collection, and the uncertainties of the sensor outputs during the periods are plotted in Figure 2, Figure 3 and Figure 4. 


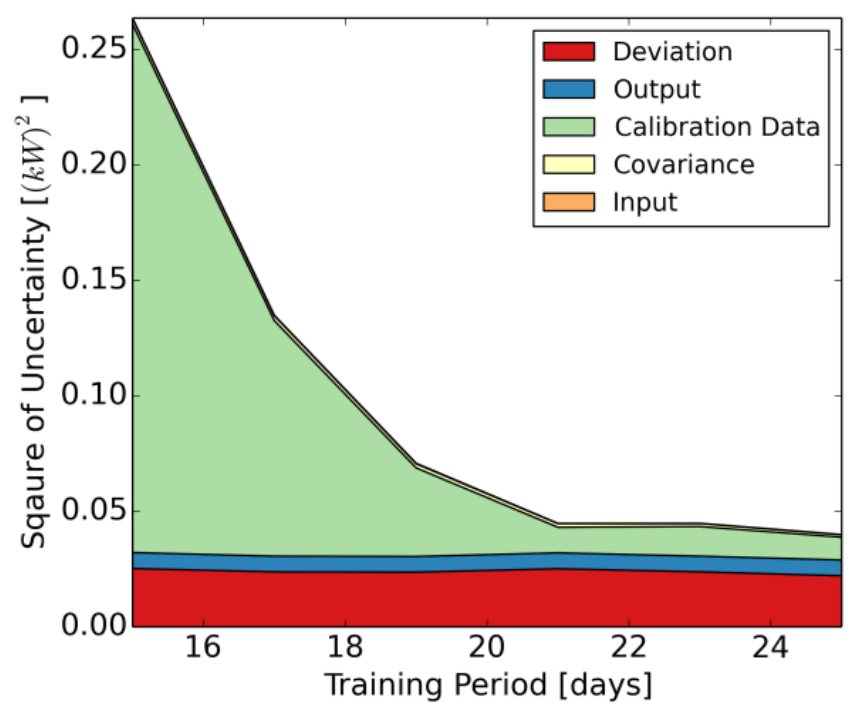

Figure 3 Square of average uncertainty of RTU3 virtual sensor output as a function of length of calibration period from July 31, 2013 


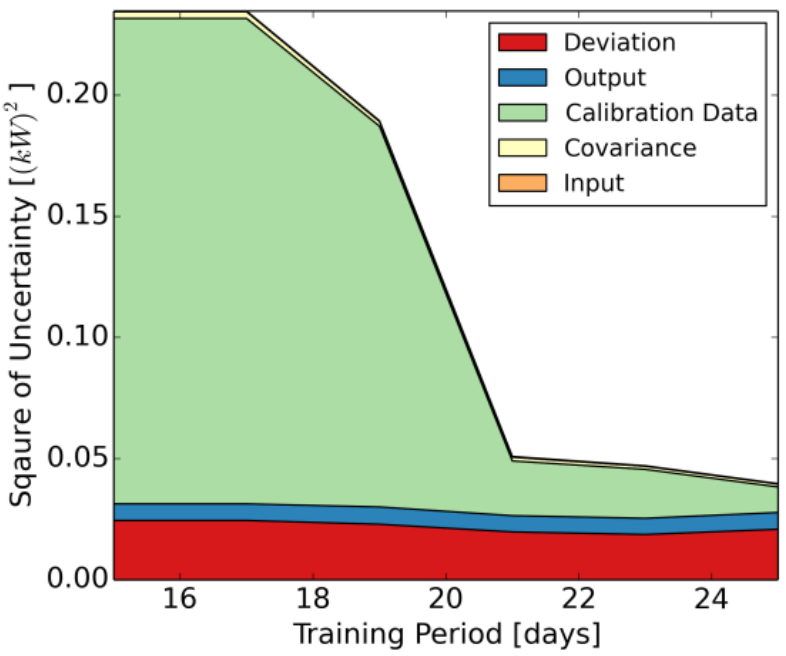

Figure 4 Square of average uncertainty of RTU4 virtual sensor output as a function of length of calibration period from July 31, 2013

Figure 2, Figure 3 and Figure 4 show that the overall uncertainty generally decreases with increasing calibration period. While increasing calibration period does not change the uncertainties from model output deviation and output measurement significantly, the uncertainty from calibration data decreases dramatically with the calibration period between 2 and 3 weeks for RTUs 3 and 4 . The increase of the calibration period reduces the overall uncertainty mainly because the root-sum-squares in Eqn. (20) decrease as the number of calibration data points increases. However, the uncertainty can also increase if the additional calibration period introduces calibration data points that are very different from the old data points. For example, in Figure 2, when the calibration period increases from 17 days to 19 days, the maximum pressure ratio across the compressor in the calibration data increases from 2.99 to 3.11. The data points corresponding to the new maximums become very significant with respect to the calibration results and this increases the uncertainty from calibration data. However, as more data points are introduced in the calibration, their significance reduces and the uncertainty component drops again.

\subsection{Change of uncertainty with inputs different from the calibration data}

To demonstrate how uncertainty of the virtual sensor output changes with different inputs, the virtual compressor and condenser fan power consumption sensors calibrated in section 3.2 were used to predict the power consumption for inputs outside the range of calibration data. Example results are tabulated in Table 6, Table 7 and Table 8.

Table 6 Prediction results for combined compressor and condenser fan power consumption virtual sensors with evaporator inlet temperature at $270 \mathrm{~K}$, condenser return bend temperature at $300 \mathrm{~K}$ and compressor suction temperature at $281 \mathrm{~K}$

\begin{tabular}{|l|r|r|r|}
\hline RTU & 2 & 3 & 4 \\
\hline
\end{tabular}


Table 7 Prediction results for combined compressor and condenser fan power consumption virtual sensors with evaporator inlet temperature at $280 \mathrm{~K}$, condenser return bend temperature at $300 \mathrm{~K}$ and compressor suction temperature at $291 \mathrm{~K}$

\begin{tabular}{|l|r|r|r|}
\hline RTU & 2 & 3 & 4 \\
\hline Estimated power consumption [kW] & 3.43 & 3.44 & 3.02 \\
\hline Relative uncertainty [\%] & 6.49 & 12.1 & 6.11 \\
\hline Relative uncertainty from output deviation [\%] & 30.7 & 12.6 & 61.0 \\
\hline Relative uncertainty from output [\%] & 10.0 & 2.3 & 12.9 \\
\hline Relative uncertainty from calibration data [\%] & 16.7 & 5.4 & 16.3 \\
\hline Relative uncertainty from model covariance [\%] & 39.8 & 78.4 & 6.9 \\
\hline Relative uncertainty from input [\%] & 2.9 & 1.2 & 2.9 \\
\hline Specific heat ratio at compressor suction & 1.28 & 1.28 & 1.34 \\
\hline Pressure ratio $P_{\text {comp,out }} / P_{\text {comp,in }}$ & 1.77 & 1.77 & 1.76 \\
\hline
\end{tabular}

Table 8 Prediction results for combined compressor and condenser fan power consumption virtual sensors with evaporator inlet temperature at $285 \mathrm{~K}$, condenser return bend temperature at $310 \mathrm{~K}$ and compressor suction temperature at $296 \mathrm{~K}$

\begin{tabular}{|l|r|r|r|}
\hline RTU & 2 & 3 & 4 \\
\hline Estimated power consumption [kW] & 4.20 & 4.34 & 3.68 \\
\hline Relative uncertainty [\%] & 6.83 & 12.3 & 5.89 \\
\hline Relative uncertainty from output deviation [\%] & 18.5 & 7.7 & 44.3 \\
\hline Relative uncertainty from output [\%] & 9.0 & 2.2 & 13.9 \\
\hline Relative uncertainty from calibration data [\%] & 19.1 & 5.2 & 21.9 \\
\hline Relative uncertainty from model covariance [\%] & 51.5 & 84.1 & 13.3 \\
\hline Relative uncertainty from input [\%] & 2.0 & 0.7 & 6.6 \\
\hline Specific heat ratio at compressor suction & 1.29 & 1.29 & 1.36 \\
\hline Pressure ratio $P_{\text {comp,out }} / P_{\text {comp,in }}$ & 1.97 & 1.97 & 1.95 \\
\hline
\end{tabular}


Table 6, Table 7 and Table 8 show that the relative uncertainties of the estimated power consumption in these cases are larger than the ones in Table 4. In particular, the relative uncertainties from model covariance in these cases are significantly higher than the ones in Table 5. For the uncertainty of the virtual sensor output at calibration data points, uncertainty from model output deviation dominates the magnitude of the uncertainty as shown in Table 5 . However, when the inputs are not identical to the calibration data, the uncertainty from model covariance dominates the magnitude of uncertainty in most cases as shown in Table 6, Table 7 and Table 8. To explain the change of the uncertainty components, the input conditions in Table 6 , Table 7 and Table 8 can be compared to the range of inputs for the calibration data points that are tabulated in Table 9.

Table 9 Maximum and minimum values of calibration data for different systems within the calibration period in section $\mathbf{3 . 3}$

\begin{tabular}{|l|r|r|r|}
\hline RTU & 2 & 3 & 4 \\
\hline Compressor suction temperature $[\mathrm{K}]$ & $276.8-287.5$ & $283.9-295.4$ & $282.8-290.0$ \\
\hline Evaporator inlet temperature $[\mathrm{K}]$ & $276.8-282.3$ & $275.1-283.9$ & $279.9-287.7$ \\
\hline Condenser return bend temperature $[\mathrm{K}]$ & $304.4-324.4$ & $311.6-335.2$ & $301.5-318.3$ \\
\hline Specific heat ratio at compressor suction & $1.279-1.313$ & $1.262-1.317$ & $1.384-1.459$ \\
\hline Pressure ratio $P_{\text {comp,out }} / P_{\text {comp,in }}$ & $2.176-3.107$ & $2.769-3.667$ & $1.797-2.212$ \\
\hline
\end{tabular}

The cases in Table 6, Table 7 and Table 8 have inputs to the virtual sensor that are outside the range of the calibration data points given in Table 9. Confidence in the model estimates is lower with these inputs, and the uncertainty from model covariance in these tables becomes higher. For example, the evaporator inlet temperature in Table 6 is $270 \mathrm{~K}$ and is lower than the minimum evaporator inlet temperature in Table 9. The uncertainties from model covariance in Table 6 become higher than that in Table 5. In the other case in Table 7 and Table 8, the pressure ratios of RTU 2 and 3 are further away from the range of pressure ratios in Table 9 than that of RTU 4, and the uncertainty from model covariance of RTU 2 and 3 in Table 7 and Table 8 is higher than that of RTU 4.

\section{Conclusions}

This paper presented a general method for uncertainty calculation of virtual sensor outputs that includes 5 separate uncertainty components: uncertainties from model output deviation, model covariance, calibration data, output measurement and input measurement. The method was demonstrated using virtual power consumption sensors calibrated with steady state data collected from 3 RTUs in the field during late summer 2013. The uncertainty method was used to identify the major sources of uncertainty and to demonstrate the effects of varying calibration periods and inputs on accuracy. In general, increasing the amount of calibration data reduces the uncertainty from calibration data significantly but does not change the uncertainties from model output deviation and output measurements. The analysis also shows that the 
uncertainty of the virtual sensor will be large if the virtual sensor is used to estimate outputs with inputs that are outside the range of inputs in the calibration data.

Since the uncertainty calculation method includes the dependence on the amount and quality of calibration data set, it can be used to assess the fitness of a calibration data set for virtual sensor calibration over the expected range of use. It can also help in quantifying the uncertainty of a virtual sensor under any set of inputs and if the virtual sensor is operating with acceptable reliability when the inputs are outside the range of calibration data.

\section{Acknowledgements}

The authors would like to acknowledge the sponsorship of the Consortium for Building Energy Innovation of the Department of Energy, Hugh Henderson for sensor installation on the packaged air conditioners and Andrew Hjortland for his collaboration on the data management for multiple projects related to the data.

\section{References}

Allus, M. A., Brereton, R. G. and Nickless, G., 1989. The Use of Experimental Design, Multilinear Regression, ANOVA, Confidence Bands and Leverage in a Study of the Influence of Metals on the Growth of Barley Seedlings, Chemom. and Intell. Labs. Syst., 6, $65-80$

ASHRAE, 2006. ANSI/ASHRAE Standard 41.1-1986 (RA 2006) Standard Method for Temperature Measurement, American Society of Heating, Refrigerating and Air-Conditioning Engineers Inc.

Byrd, R. H., Lu, P., Nocedal, J. and Zhu, C., 1995. A Limited Memory Algorithm for Bound Constrained Optimization, SIAM J. on Sci. and Stat. Comput., 16:5, 1190 - 1208

DOE, 2007. 2007 Buildings Energy Databook, http://buildingsdatabook.eren.doe.gov/docs/6.2.5.pdf, Department of Energy, Accessed on 2013/10/07.

EIA, 2003. 2003 Commercial Building Energy Consumption and Expenditures, http://www.eia.doe.gov/emeu/cbecs/cbecs2003/public use 2003/cbecs pudata2003.html. , Energy Information Administration, Accessed on 2013/10/07.

Graybill, F. A. and Iyer, H. K., 1994. Regression Analysis: Concepts and Applications, $1^{\text {st }}$ Edition, Duxbury $\operatorname{Pr}$

Hendricks, W. A. and Robey, K. W., 1936. The Sampling Distribution of the Coefficient of Variation, Ann. of Math. Stat., 7:3, 129 - 132

Jähnig, D. I., Reindl, D. T., Klein, S. A., 2000, A semi-empirical method for representing domestic refrigerator/freezer compressor calorimeter test data. ASHRAE Trans., 106, 112 - 130

Kesteven, G. L., 1946. The Coefficient of Variation, Nat., 158, 520 - 521 
Kim, W. and Braun, J. E., 2012. Performance evaluation of a virtual refrigerant charge sensor, Int. J. of Refrig., 36, 1130 - 1141

Kline, S. J. and McClinktock, F. A., 1953. Describing uncertainties in single-sampled experiments, Mech. Eng., 75:1, 3-8

Laurent, R. T. ST. and Cook, R. D., 1993. Leverage, Local Influence and Curvature in nonlinear regression. Biom., 80:1, 99 - 106

Li, H. and Braun, J. E., 2007, Decoupling Features and Virtual Sensors for Diagnosis of Faults in Vapor Compression Air Conditioners, Int. J. of Refrig., 30:3, 546-564

Li, H. and Braun, J. E., 2009. Virtual Refrigerant Pressure Sensors for Use in Monitoring and Fault Diagnosis of Vapor-Compression Equipment, HVAC\&R Res., 15:3, 597-616

Li, H., Yu, D. and Braun, J. E., 2011. A review of virtual sensing technology and application in building systems, HVAC\&R Res., 17:5, 619-645

Moffat, R. J., 1988. Describing Uncertainties in Experimental Results, Exp. Therm. and Fluid Sci., $1,3-17$

Montgomery, D. C., 2005. Design and Analysis of Experiments, $6^{\text {th }}$ Edition, Jon Wiley \& Sons Nocedal, J. and Wright, S. J., 2000. Numerical Optimization, $2^{\text {nd }}$ Edition, Springer

Song, L., Joo, I. and Wang, G., 2012. Uncertainty analysis of a virtual water flow measurement in building energy consumption monitoring, HVAC\&R Res., 18:5, 997-1010

Song, L., Wang, G. and Brambley, M. R., 2011. Uncertainty analysis for a virtual flow meter using an air-handling unit chilled water valve, HVAC\&R Res., 19:3, 335-345

Taylor, B. N. and Thompson, A., 2008. The International System of Units (SI) (Special Publication 330), Gaithersburg, MD: National Institute of Standards and Technology

Yu, D., Li, H., Yu, Y. and Xiong, Jun, 2011a. Virtual calibration of a supply air temperature sensor in rooftop air conditioning units, HVAC\&R Res., 17:1, 31-5

Yu, D., Li, H. and Yang, M., 2011b. A virtual supply airflow rate meter for rooftop air-conditioning units, Build. and Environ., 46, 1292-1302

Zhao, X., Yang, M. and Li, H., 2012. A virtual condenser fouling sensor for chillers, Energy and Build., 52, 68-76

\section{Appendix A: Evaporator fan power virtual sensor model and uncertainty}


The virtual fan power sensor provides an estimate of the true value at any time instant during the fan operation. For this application, the evaporator fans operate at a single speed and the population mean of the steady state fan power consumption can be expressed using the following equation as an unbiased estimate of the true value of the evaporator fan power consumption at any time instant.

$$
\dot{W}_{\text {evap }, \text { true }}=\mu\left(\dot{W}_{\text {evap }}\right)+\varepsilon
$$

The virtual sensor predicts the evaporator fan power consumption using the sample mean of the fan power consumption from the calibration data as

$$
\hat{\dot{W}}_{\text {evap }}=\overline{\dot{W}}_{\text {evap,train }}
$$

Estimates of average evaporator fan power consumption were extracted from the field data for each RTU assuming that the fans reach steady state operation instantaneously and by the averaging the data for periods where only the evaporator fan operated.

Comparing Eqn. (32) with Eqn. (6), the model output $y$ of the virtual sensor is $\dot{W}_{\text {evap }}$ with a model input vector $\vec{x}$ of 1 and a model coefficient vector $\vec{\beta}$ of $\overline{\dot{W}}_{\text {evap }}$. Since the model input vector $\vec{x}$ is a constant value of 1 and has zero uncertainty, the entries in $\mathbf{J}_{\text {train }}$ and $\frac{\partial f}{\partial \vec{\beta}}$ in Eqn. (18) are one, the second order derivatives in Eqn. (13) are zero and the uncertainty due to input uncertainty is zero. Using a $95 \%$ confidence interval to calculate the uncertainty, the uncertainties from model output deviation and model covariance are given as follows.

$$
\begin{gathered}
\Delta \hat{\hat{W}}_{\text {evap }, \text { dev }}=t_{n-1,0.95} \sigma_{\dot{W}_{\text {evap }}} \\
\Delta \hat{\dot{W}}_{\text {evap }, \text { cov }}=\frac{t_{n-1,0.95} \sigma_{\dot{W}_{\text {evap }}}}{n}
\end{gathered}
$$

The uncertainty due to calibration data uncertainty in Eqn. (20) leads to the following for this case.

$$
\Delta \hat{\dot{W}}_{\text {evap }, \text { train }}=\frac{\sum_{i}\left(\Delta \dot{W}_{\text {evap,train }, i}\right)}{n}
$$

The uncertainty due to output measurement uncertainty in Eqn. (23) leads to 


$$
\Delta \hat{\dot{W}}_{\text {evap,output }}=\frac{\hat{\dot{W}}_{\text {evap }}}{n} \sum_{i}\left(\frac{\Delta \dot{W}_{\text {evap }, \text { train }, i}}{\dot{W}_{\text {evap }, \text { train }, i}}\right)
$$

The overall uncertainty of the virtual sensor output is calculated by taking the sum of squares of the uncertainties in Eqn. (24) to yield

$$
\Delta \hat{\dot{W}}_{\text {evap }}=\sqrt{\frac{\sum_{i}\left(\Delta \dot{W}_{\text {evap }, \text { train }, i}\right)^{2}}{n^{2}}+\left(1+\frac{1}{n^{2}}\right)\left(t_{n-1,0.95} \sigma_{\dot{W}_{\text {evap }}}\right)^{2}+\left(\frac{\hat{\dot{W}}_{\text {evap }}}{n} \sum_{i} \frac{\left.\Delta \dot{W}_{\text {evap }, \text { train }, i}\right)^{2}}{\dot{W}_{\text {evap }, \text { train }, i}}\right.}
$$

\section{Appendix B: Uncertainty calculation of virtual sensor of compressor and condenser fan power consumption}

Since the compressor and condenser fan operate together for the RTUs and only the total energy consumption was measured, a single virtual sensor was constructed for each RTU to estimate the combined power consumption. The mathematical model was formed by modifying a reciprocating compressor model (Jähnig et al. 2000).

$$
\dot{W}_{\text {comp }+ \text { cond }}=\frac{k P_{\text {comp }, \text { in }}}{k-1} \frac{\left[\beta_{\text {comp }, 1}-\beta_{\text {comp }, 2}\left(\left(\frac{P_{\text {comp }, \text { out }}}{P_{\text {comp }, \text { in }}}\right)^{\frac{1}{k}}-1\right)\right]\left(\left(\frac{P_{\text {comp }, \text { out }}}{P_{\text {comp }, \text { in }}}\right)\left(\frac{k-1}{k}\right)-1\right)}{\left(1+\beta_{\text {comp }, 3}\left(P_{\text {comp }, \text { in }}-\beta_{\text {comp }, 4}\right)\right)\left(1+\beta_{\text {comp }, 5}\left(P_{\text {comp }, \text { out }}-\beta_{\text {comp }, 6}\right)\right)}
$$

This model is different from the model in Jähnig et al. (2000) because it includes the effect of both compressor suction and discharge pressure on compressor efficiency whereas the original model estimates the efficiency as a function of compressor suction pressure only. The pressures in this model are estimated using virtual pressure sensors that calculate the refrigerant saturated vapor pressures at the condenser return bend temperature and the evaporator inlet temperature using the following equations (Li and Braun 2009).

$$
\begin{gathered}
P_{\text {comp }, \text { out }}=P_{v}\left(T=T_{\text {cond }}\right) \\
P_{\text {comp }, \text { in }}=P_{v}\left(T=T_{\text {evap }}\right)
\end{gathered}
$$

The specific heat ratio $k$ is calculated using the following equation with the measured compressor inlet temperature and the virtual compressor inlet pressure.

$$
k=\frac{c_{p}\left(P=P_{\text {comp }, \text { in }}, T=T_{\text {comp }, \text { in }}\right)}{c_{v}\left(P=P_{\text {comp }, \text { in }}, T=T_{\text {comp }, \text { in }}\right)}
$$


The combined compressor and condenser fan power model can be written in the form of Eqn. (11) as

$$
\dot{W}_{\text {comp }+ \text { cond,true }}=f_{\text {comp }+ \text { cond }}\left(\vec{x}_{\text {true }}=\left[\begin{array}{c}
T_{\text {comp }, \text { in }, \text { true }} \\
T_{\text {cond,true }} \\
T_{\text {evap }, \text { true }}
\end{array}\right], \vec{\beta}_{\text {comp }, \text { true }}\right)+\varepsilon
$$

The prediction equation for the virtual power consumption becomes

$$
\hat{\dot{W}}_{\text {comp }+ \text { cond }, \text { true }}=f_{\text {comp }+ \text { cond }}\left(\vec{x}=\left[\begin{array}{c}
T_{\text {comp }, \text { in }} \\
T_{\text {cond }} \\
T_{\text {evap }}
\end{array}\right], \overrightarrow{\hat{\beta}}_{\text {comp }}\right)
$$

To provide data for estimating the coefficients in the model, steady state data for combined compressor and condenser fan operation were obtained as described in section 3 . The coefficients in compressor model were estimated by minimizing the following objective function using the L-BFGS method (Byrd et al. 1995).

$$
\begin{aligned}
& \theta\left(\hat{\beta}_{\text {comp }, 1}, \hat{\beta}_{\text {comp }, 2}, \hat{\beta}_{\text {comp }, 3}, \hat{\beta}_{\text {comp }, 4}, \hat{\beta}_{\text {comp }, 5}, \hat{\beta}_{\text {comp }, 6}\right) \\
& =\left(\hat{\dot{W}}_{\text {comp }+ \text { cond }}\left(\vec{x}_{i}=\left[\begin{array}{c}
\bar{T}_{\text {comp }, i n, i} \\
\bar{T}_{\text {cond }, i} \\
\bar{T}_{\text {evap }, i}
\end{array}\right], \overrightarrow{\hat{\beta}}_{\text {comp }}\right)-\dot{W}_{\text {comp }+ \text { cond }, i}\right)^{2}
\end{aligned}
$$

Assuming a 95\% confidence interval, the uncertainty of the predicted power consumption $\widehat{\dot{W}}_{\text {comp }+ \text { cond }}$ is calculated by Eqns. (47), (48), (49), (50) and (51). 


$$
\begin{aligned}
& \left(\Delta \hat{\dot{W}}_{\text {comp }+ \text { cond }}\left(\vec{x}, \overrightarrow{\hat{\beta}}_{\text {comp }}\right)\right)^{2} \\
& =\Sigma_{l} \Sigma_{k}\left(\left(\Sigma_{p} \frac{\partial \hat{\hat{W}}_{\text {comp }+ \text { cond }}\left(\vec{x}, \overrightarrow{\hat{\beta}}_{\text {comp }}\right)}{\partial \hat{\beta}_{\text {comp }, p}} \frac{\partial \hat{\beta}_{\text {comp }, p}}{\partial x_{\text {train }, l k}}\right) \Delta x_{\text {train }, l k}\right)^{2} \\
& +\Sigma_{k}\left(\left(\Sigma_{p} \frac{\partial \hat{\dot{W}}_{\text {comp }+ \text { cond }}\left(\vec{x}, \overrightarrow{\hat{\beta}}_{\text {comp }}\right)}{\partial \hat{\beta}_{\text {comp }, p}} \frac{\partial \hat{\beta}_{\text {comp }, p}}{\partial \dot{W}_{\text {comp }+ \text { cond }, \text { train }, k}}\right) \Delta \dot{W}_{\text {comp }+ \text { cond }, \text { train }, k}\right)^{2}+t_{n-m, 0.95}^{2} \sigma_{w x}^{2} \\
& +t_{n-m, 0.95}^{2} \sigma_{w x}^{2}\left(( \frac { \partial \hat { \hat { W } } _ { \text { comp } + \text { cond } } ( \vec { x } , \vec { \hat { \beta } } _ { \text { comp } } ) } { \partial \vec { \beta } _ { \text { comp } } } ) ^ { T } \left(\mathbf{J}_{w, \text { train }}^{T} \mathbf{J}_{w, \text { train }}\right.\right. \\
& \left.\left.-\left[\overrightarrow{\hat{W}}_{\text {comp }+ \text { cond,train }}-\overrightarrow{\hat{\dot{W}}}_{\text {comp }+ \text { cond,train }}\right]\left[\mathbf{H}_{w, \text { train }}\right]\right)^{-1}\left(\frac{\partial \hat{\dot{W}}_{\text {comp }+ \text { cond }}\left(\vec{x}, \overrightarrow{\hat{\beta}}_{\text {comp }}\right)}{\partial \vec{\beta}_{\text {comp }}}\right)\right)
\end{aligned}
$$

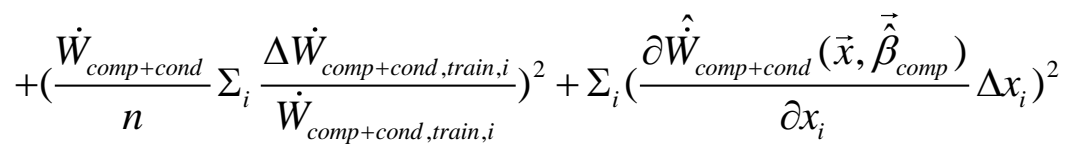

$$
\begin{aligned}
& \mathbf{J}_{w, \text { train }}=\left[\begin{array}{ccc}
\frac{\partial \hat{\dot{W}}_{\text {comp }+ \text { cond }}\left(\vec{x}_{\text {train }, 1}, \overrightarrow{\hat{\beta}}_{\text {comp }}\right)}{\partial \hat{\beta}_{\text {comp }, 1}} & \cdots & \frac{\partial \hat{\dot{W}}_{\text {comp }+ \text { cond }}\left(\vec{x}_{\text {train }, 1}, \overrightarrow{\hat{\beta}}_{\text {comp }}\right)}{\partial \hat{\beta}_{\text {comp }, 6}} \\
\vdots & \cdots & \vdots \\
\frac{\partial \hat{\hat{W}}_{\text {comp }+ \text { cond }}\left(\vec{x}_{\text {train }, n}, \overrightarrow{\hat{\beta}}_{\text {comp }}\right)}{\partial \hat{\beta}_{\text {comp }, 1}} & \cdots & \frac{\partial \hat{\dot{W}}_{\text {comp }+ \text { cond }}\left(\vec{x}_{\text {train }, n}, \overrightarrow{\hat{\beta}}_{\text {comp }}\right)}{\partial \hat{\beta}_{\text {comp }, 6}}
\end{array}\right] \\
& \mathbf{H}_{w, \text { rain }, i}=\left[\begin{array}{ccc}
\frac{\partial^{2} \hat{\dot{W}}_{\text {comp }+ \text { cond }}\left(\vec{x}_{\text {train }, i}, \overrightarrow{\hat{\beta}}\right)}{\partial \hat{\beta}_{\text {comp }, 1}^{2}} & \ldots & \frac{\partial^{2} \hat{\dot{W}}_{\text {comp }+ \text { cond }}\left(\vec{x}_{\text {train }, i}, \overrightarrow{\hat{\beta}}\right)}{\partial \hat{\beta}_{6} \partial \hat{\beta}_{1}} \\
\vdots & \frac{\partial^{2} \hat{\dot{W}}_{\text {comp }+ \text { cond }}\left(\vec{x}_{\text {train }, i}, \overrightarrow{\hat{\beta}}\right)}{\partial \hat{\beta}_{j} \delta \hat{\beta}_{k}} & \vdots \\
\frac{\partial^{2} \hat{\hat{W}}_{\text {comp }+ \text { cond }}\left(\vec{x}_{\text {train }, i}, \overrightarrow{\hat{\beta}}\right)}{\partial \hat{\beta}_{\text {comp }, 1} \partial \hat{\beta}_{\text {comp }, 6}} & \ldots & \frac{\partial^{2} \hat{\dot{W}}_{\text {comp }+ \text { cond }}\left(\vec{x}_{\text {train }, i}, \overrightarrow{\hat{\beta}}\right)}{\partial \hat{\beta}_{6}{ }^{2}}
\end{array}\right] \\
& {\left[\overrightarrow{\dot{W}}_{\text {comp }+ \text { cond }, \text { train }}-\overrightarrow{\hat{\dot{W}}}_{\text {comp }+ \text { cond }, \text { train }}\right]\left[\mathbf{H}_{w, \text { train }}\right]=\Sigma_{i}\left(\dot{W}_{\text {comp }+ \text { cond }, \text { train }, i}-\hat{\dot{W}}_{\text {comp }+ \text { cond }, \text { train }, i}\right) \mathbf{H}_{w, \text { train }, i}} \\
& \sigma_{w x}^{2}=\frac{\sum_{i}\left(\hat{\dot{W}}_{\text {comp }+ \text { cond,train, } i}\left(\vec{x}_{\text {train }, i}, \overrightarrow{\hat{\beta}}_{\text {comp }}\right)-\dot{W}_{\text {comp }+ \text { cond }, \text { train }, i}\right)^{2}}{n-m}
\end{aligned}
$$

For simplicity, the derivation of Eqn. (47) assumed that the refrigerant property calculation equations are true models and their only source of uncertainty is the uncertainty in the inputs. The derivatives of the coefficients with respect to other variables in Eqn. (47) cannot be derived analytically and are approximated by finite differences. The derivatives related to calibration 
data, the uncertainties from calibration data, the mean square error, the t-statistics, the Jacobian leverage and the uncertainty from outputs are constants in Eqn. (47). They remain unchanged in the uncertainty calculation of the virtual sensor output after calibration. 